\title{
Combined imaging and analytical STEM of ultra-thin cuprate films
}

Vesna Srot $^{1}$, Yi Wang ${ }^{1}$, Matteo Minola ${ }^{1}$, Ute Salzberger ${ }^{1}$, Marco Salluzzo ${ }^{2,3}$, Gabriella Maria De Luca $^{3,2}$, Bernhard Keimer ${ }^{1}$ and Peter A. van Aken ${ }^{1}$

${ }^{1 .}$ Max Planck Institute for Solid State Research, Stuttgart, Germany

2. CNR-SPIN Napoli Complesso Monte Sant' Angelo via Cinthia, Napoli, Italy

3. Dipartimento di Fisica “E. Pancini" Complesso Monte Sant' Angelo via Cinthia, Napoli, Italy

* Corresponding author: V.Srot@fkf.mpg.de

Considerable advances in scanning transmission electron microscopy (STEM) have revealed fascinating phenomena in functional complex oxide materials at the atomic scale. Simultaneous acquisition of annular bright-field (ABF) and high-angle annular dark-field (HAADF) STEM images opened up an elegant path for combined imaging of light and heavy elements. Furthermore, combining STEM imaging with advanced analytical methods, such as electron energy-loss spectroscopy (EELS) and energy-dispersive X-ray spectroscopy (EDX), sets an exceptionally powerful approach to study very thin films.

In this work, high quality $\mathrm{NdBa}_{2} \mathrm{Cu}_{3} \mathrm{O}_{7}$ (NBCO) ultra-thin films (1, 2 and 4 unit cells) have been deposited on $\mathrm{TiO}_{2}$-terminated $\mathrm{SrTiO}_{3}$ (STO) substrates by high oxygen pressure diode sputtering [1]. The layer stacking sequence along the growth direction parallel to the $c$-axis of $\mathrm{NBCO}$ is $\mathrm{BaO}_{-} \mathrm{CuO}_{2}-$ $\mathrm{Nd}-\mathrm{CuO}_{2}-\mathrm{BaO}-\mathrm{CuO}$. In $\mathrm{NBCO}$, the $\mathrm{Cu}$ atoms are located both in the $\mathrm{CuO}_{2}$ planes and in the $\mathrm{CuO}$ chains that run parallel to the planes and structurally separate the superconducting $\mathrm{CuO}_{2}$ bilayers. $\mathrm{Nd}$ atoms are placed within each bilayer in-between the two $\mathrm{CuO}_{2}$ planes. $\mathrm{Ba}$ atoms are located between the planes and the chains. Major changes of the NBCO's physical properties can appear due to oxygen content fluctuations [2]. For non-stoichiometric $\mathrm{NdBa}_{2} \mathrm{Cu}_{3} \mathrm{O}_{7-\mathrm{x}}, \mathrm{x}$ denotes the amount of vacancies present in the $\mathrm{CuO}$ chains. The charge balance between the $\mathrm{CuO}_{2}$ planes and $\mathrm{CuO}$ chains affects and effectively dictates $T_{\mathrm{c}}$ [3].

We have employed atomic-column resolved quantitative STEM imaging combined with forefront analytical techniques to investigate the ultra-thin NBCO films by using an advanced aberrationcorrected JEOL JEM-ARM200F microscope equipped with a DCOR probe corrector operated at 200 $\mathrm{kV}$. Simultaneously acquired and overlaid HAADF- and ABF-STEM images of a selected NBCO ultra-thin film on STO (top) and an ABF-STEM image with the superimposed NBCO structural model (bottom) are shown in Figure 1. The improvement of the signal-to-noise ratio as well as the reduction of the image distortions was achieved by multiple frame acquisition [4]. Using such approach, we were able to quantitatively analyse the local cation and anion sub-lattices. In addition, we have performed STEM image simulations in order to correlate the lowest detectable oxygen concentrations with the sample thickness.

The chemical identity of ultra-thin NBCO films and their interfaces with the STO substrates were studied by combining atomically resolved ADF-STEM imaging with EELS and EDX mapping. An EELS elemental map obtained from a representative NBCO/STO interface is shown in Figure 2. Chemical maps have confirmed that the STO substrate is $\mathrm{TiO}_{2}$ terminated. Furthermore, surface steps detected on the STO substrate and consequent defects in NBCO films were studied and will be discussed [5]. 
References:

[1] M Salluzzo et al., Phys rev B 78 (2008), 054524.

[2] H Shaked et al., Phys Rev B 41 (1990), p. 4173.

[3] RJ Cava et al., Physica C 165 (1990), p. 419.

[4] Y Wang et al., Ultramicroscopy 168 (2016), p. 46.

[5] This project has received funding from the European Union's Horizon 2020 research and innovation programme under grant agreement No. 823717 - ESTEEM3.

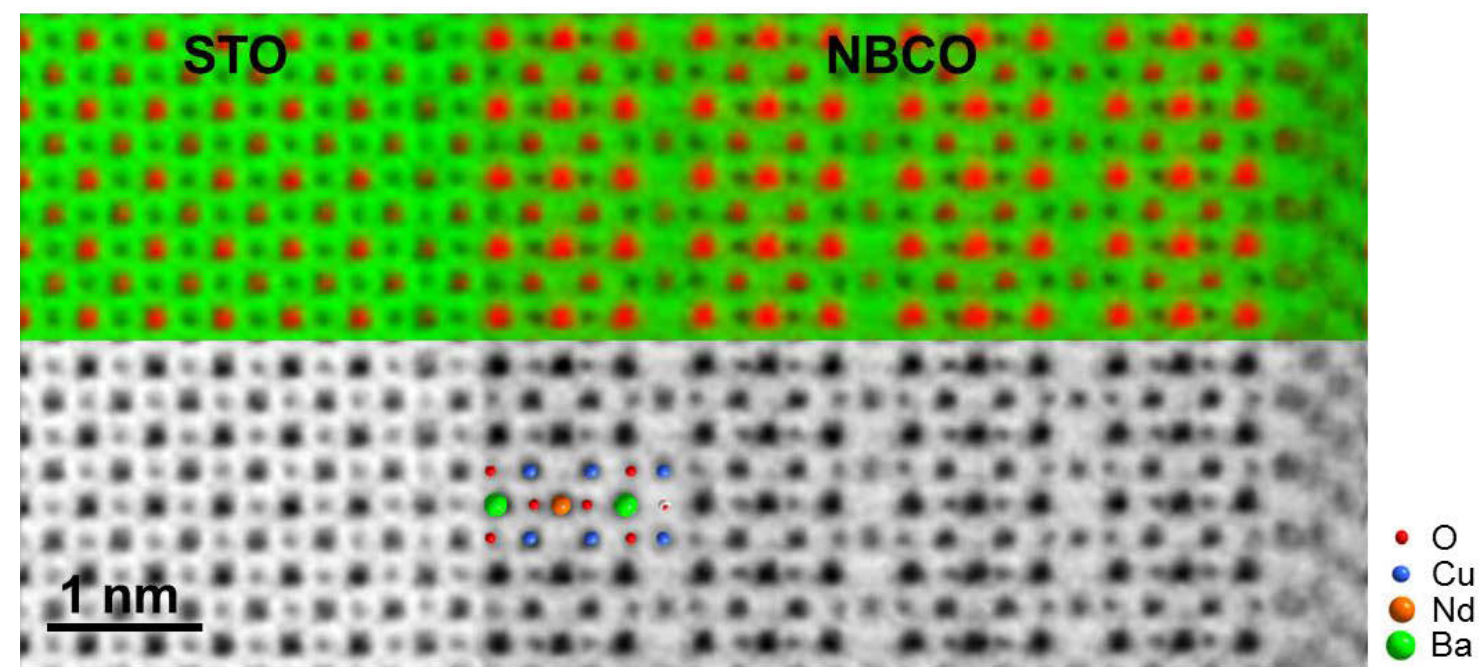

Figure 1. Overlay of simultaneously acquired HAADF- (red) and ABF- (green) STEM images (top) of a NBCO ultra-thin film (4 unit cells) grown on STO and ABF-STEM image (bottom) with an overlay of the NBCO structural model.
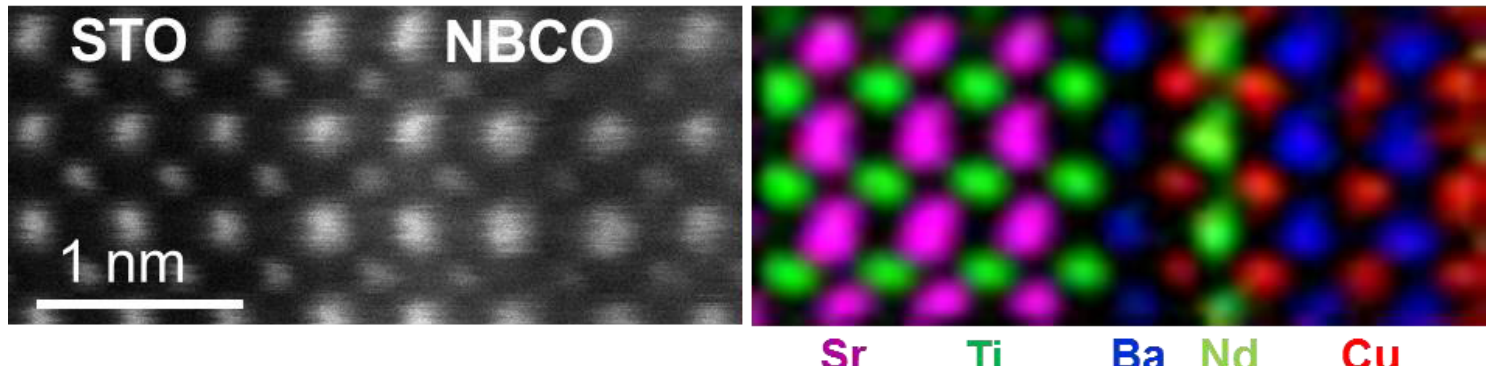

Figure 2. ADF-STEM image of the NBCO/STO interface with a corresponding atomically resolved EELS elemental map. 